\title{
THE MERAN MEETING OF THE DEUTSCHE MATHEMATIKER-VEREINIGUNG.
}

The annual meeting of the Deutsche Mathematiker-Vereinigung was held, in affiliation with the seventy-seventh regular convention of the Deutsche Naturforscher und Aerzte, at Meran, in the Tyrol, September 24-30, 1905. Eighty persons attended the sessions, which were presided over by Professors Köhler, Stäckel, Czuber, Geiser and Hensel respectively. The following papers were read:

(1) Professor Czuber, Vienna: "The question of introducing the calculus into the preparatory schools, from an Austrian standpoint (Report)."

(2) Professor Hočevar, Graz: "Should the calculus be introduced into the preparatory schools, or not?"

(3) Professor W ̈̈̈LSCH, Brünn : "Geometric-mechanic pictures of a new binary-invariant writing of chemical formulas."

(4) Professor Henz, Vienna: "The year of Christ's birth."

(5) Professor Wien, Würzburg: "Report on the partial differential equations of physics."

(6) Professor Hasenöhrl, Vienna: "On methods of integration of Maxwell equations for electric vibrations."

(7) Professor Gans, Tübingen : "Gravitation and electromagnetism."

(8) Professor Wirtinger, Vienna : "On branching of functions of two variables."

(9) Dr. Koebe, Berlin : "On conformal representation of multiply connected regions, particularly of regions that are bounded by arcs of circles."

(10) Professor Levi-Crvita, Padua : "On a technical problem in relation to conformal representation."

(11) Professor Schonnflies, Königsberg: "On the socalled paradoxes of the theory of aggregates."

(12) Professor Hensel, Marburg: "On the arithmetic properties of algebraic and of transcendental numbers."

(13) Professor Zindien, Innsbruck : "Report on the development of differential line geometry."

(14) Professor Epstein, Strassburg : "Report on new investigations of the Riemann s-function and its extensions."

(15) Professor KoHN, Vienna: "On doubly apolar quad-

(16) Professor Schlesinger, Clausenburg: "On a representation of the system of absolute geometry." 
(17) Dr. Grünwald, Vienna : "Certain geometric applications of dual numbers."

(18) Professor MüLlen, Vienna : "Contributions to cyclography."

(19) Professor MüLler, Vienna: "Descriptive geometry as an illustration of abstract projective geometry."

(20) Professor DokuliL, Vienna : "Photogrammetry in the service of the history of art."

(21) Professor DokuliL, Vienna : "Construction and study of stereoscopic pictures."

(22) Dr. FeJÉ, Clausenburg : "On equilibrium in a resisting medium."

(23) Professor Stäckel, Hanover : "Pairs of isometric surfaces."

Abstracts of the papers, so far as obtainable, follow. They are numbered to correspond to the titles in the list above.

1. Austrian schools give more time to mathematics and the sciences than is allowed in Germany, but Professor Czuber urges that the principles of the theory of functions be introduced empirically much earlier than at present. The idea of a derivative and of a definite integral could be taught in the gymnasia without lengthening the time devoted to mathematics. In conclusion he stated exactly formulated proposals as to the manner of carrying out the reform. The hearers, with one exception, agreed heartily with Professor Czuber's views.

2. Professor Hocevar answers his question in the affirmative and further discusses the plan outlined in 1 .

5. This report will appear in full in the Jahresbericht.

6. Professor Hasenöhrl's paper shows how to apply certain methods of the theory of potential and related subjects to the integration of Maxwell's equations.

7. Professor Gans developed the Lorentz idea, that electrons of the same sign repel each other rather less strongly than those of opposite sign attract each other. A conductor may give no insulation for gravitation, yet furnish complete insulation for electrostatic forces. The deviation of this law of attraction from Newton's law depends only on quantities of the second 
order. The consequences of this deviation should be investigated.

8. After giving a detailed discussion of the branching of the function $z^{3}+3 x z+2 y=0$ at $x=0, y=0$, Professor Wirtinger offered various considerations of the branching of a Riemann space, and interpreted the results by analysis situs.

9. Dr. Koebe proved the following theorem : If $\tau, \tau^{\prime}$ be two smooth plane regions, each bounded by $\rho+1$ circles, of which no two cut or touch each other, and if $\tau$ can be mapped conformally upon $\tau^{\prime}$, then the analytic function by means of which the representation is effected must be an integral or fractional linear function, and extended the result to apply to certain Riemann surfaces.

10. A problem in technical electricity was shown to depend upon the conformal representation of a multiply connected region bounded by circles. That discussed by Professor Levi-Civita was the determination of the most advantageous arrangement of the auxiliary conductor in the Wurtz lightning conductor.

11. This paper is to appear in the Jahresbericht.

13. The full report of which the present paper forms a part will be published in the Jahresbericht.

14. After speaking of the properties of Riemann's zeta functions, Dr. Epstein divided the generalizations into two groups, first, that of Dedekind, investigated by Landau, which relates to algebraic numbers, and second that studied by Lipschitz, Hurwitz and others, which bears the same relation to Riemann's zeta function as theta functions of $p$ variables do to elliptic theta functions.

15. Professor Kohn showed that two quadric surfaces would be doubly apolar when one contains two generators of the same system which are polar reciprocal as to the other. Each surface has then a pair of lines reciprocal as to the other, in each generation. The quadrilaterals formed by these generators were then investigated. 
16. The subject matter of Professor Schlesinger's paper will be incorporated in his forthcoming book on absolute geometry.

17. Professor Grünwald applies the numbers $\mu+\epsilon \nu\left(\epsilon^{2}=0\right)$ to the study of directed lines in space.

23. From every pair of applicable surfaces it is possible to derive twenty-eight pairs of applicable surfaces. Professor Stäckel's paper appeared in full in the Jahresbericht, volume 14, pages 507-516.

E. A. Miller, EliJah Swift.

\title{
THE PRESENT AND THE FUTURE OF MATHE- MATICAL PHYSICS.*
}

\author{
ADDRESS DELIVERED BEFORE THE SECTION OF APPLIED \\ MATHEMATICS OF THE INTERNATIONAL CON- \\ GRESS OF ARTS AND SCIENCE, ST. LOUIS, \\ SEPTEMBER 24, 1904.
}

BY PROFESSOR H. POINCARÉ.

WhAT is the present state of mathematical physics? What are its problems? What is its future? Is it about to change its orientation? Will the object and methods of this science appear in ten years to our immediate successors in the same light as they appear to us? Or are we to witness a far-reaching transformation? These are the questions we are forced to face to-day at the outset of our inquiry.

It is easy to ask; difficult to answer. If we felt tempted to hazard a prediction, we should easily resist this temptation by stopping to think of the nonsense the most eminent scholars of a hundred years ago would have spoken in answer to the question of what this science would be in the nineteenth century. They would have thought themselves bold in their predictions; and after the event how timid we should have found them! Do not expect of me therefore any kind of prophesy.

But if, like all prudent physicians, I refuse to give a prognosis, still I cannot deny myself a little diagnosis. Well, then, yes; there are symptoms of a serious crisis, which would seem

\footnotetext{
* Translated with the author's permission by Professor J. W. Young.
} 